\title{
MINIMAL SLIT REGIONS AND LINEAR OPERATOR METHOD
}

\author{
By Kôtaro OiKawa
}

1. Let $\Omega$ be a plane region containing the point at infinity. Let $\widetilde{F}_{\Omega}$ be the family of all the univalent functions $f$ on $\Omega$ having the expansion

$$
f(z)=z+\frac{c}{z}+\cdots
$$

about $\infty$. The function maximizing (minimizing) $\operatorname{Re} c$ in $\mathfrak{f}_{\Omega}$ exists and is determined uniquely, which we denote by $\varphi_{\Omega}\left(\psi_{\Omega}\right.$, resp.).

The image region $\phi_{\Omega}(\Omega)\left(\phi_{\Omega}(\Omega)\right)$ is a horizontal (vertical) parallel slit plane. Conversely, however, an arbitrary horizontal (vertical) parallel slit plane can not be, in general, the image of an $\Omega$ under $\varphi_{\Omega}\left(\psi_{\Omega}\right)$; in fact the measure of $\varphi_{\Omega}(\Omega)^{c}$ and $\phi_{\Omega}(\Omega)^{c}$ vanish. Accordingly, with Koebe, we introduce the following:

Definition. A horizontal (vertical) parallel slit plane $\Delta$ is said to be minimal if $\Delta=\varphi_{\Omega}(\Omega)\left(\Delta=\phi_{\Omega}(\Omega)\right.$, resp.) for an $\Omega$ containing $\infty$.

The minimality of slit regions is characterized by moduli of quadrilaterals (Grötzsch [2]) or extremal length (Jenkins [3]). From the point of view of the latter a number of interesting properties are derived in Suita's paper in these Reports [8].

The linear operator method due to Sario [6] (see also Chapter III of the book by Ahlfors-Sario [1]) gives us another approach to $\varphi_{\Omega}$ and $\phi_{\Omega}$. From this a characterization of minimality is derived, which is rather similar to the original one due to Koebe [4]. It is the purpose of the present paper to show how to use this method to prove alternatively a part of Suita's results mentioned above.

2. We begin with reviewing the definition of the normal linear operators $L_{0}$ and $L_{1}$ in Ahlfors-Sario [1].

Let $W$ be an open Riemann surface, let $V$ be a regularly imbedded non-compact subregion with compact relative boundary $\alpha$. For any real analytic function $f$ on $\alpha$, consider the problem of constructing the function $u$ such that

$$
\text { harmonic on } V \cup \alpha, \quad u=f \text { on } \alpha \text {. }
$$

If $V$ is the interior of a compact bordered surface we can assign the behavior of $u$ on $\beta=$ (border of $V$ ) $-\alpha$ so that $u$ may be determined uniquely. For our purpose the following two are necessary:

Received March 22, 1965. 


$$
\begin{aligned}
& \left(L_{0}\right): \quad d u^{*}=0 \text { along } \beta, \\
& \left(L_{1}\right): \quad d u=0 \text { along } \beta, \quad \int d u^{*}=0 \text { for each contour of } \beta ;
\end{aligned}
$$

here the correspondence $f \rightarrow u$ is expressed by the notations in the left.

Note that the present $L_{1}$ is the $(P) L_{1}$ in Ahlfors-Sario's book with respect to the canonical partition $P$. (See [1, p. 160].)

If $V$ is arbitrary we may define $L_{0}$ and $L_{1}$ as the limit through an exhaustion. We can define them also as follows:

Definition. $L_{0} f$ is defined as the $u$ determined uniquely by the condition (2), $D_{V}(u)<\infty$, and

$$
\int_{V}(d u)(d v)^{*}=\int_{\alpha} v d u^{*}
$$

for every harmonic function $v$ on $\bar{V}$ with $D_{V}(v)<\infty$. $L_{1} f$ is defined as the $u$ determined uniquely by the condition $(2), D_{V}(u)<\infty, \int_{r} d u^{*}=0$ for every dividing cycle $\gamma$ which does not separate components of $\alpha$, and

$$
\int_{V}(d u) \omega=\int_{\alpha} f \omega
$$

for every harmonic differential $\omega$ on $V \cup \alpha$ such that $\|\omega\|_{V}<\infty$ and $\int_{\gamma} \omega=0$ for every $\gamma$ mentioned above.

We remark the following:

(i) If $V$ is the interior of a compact bordered surface, this definition coincides with the previous.

(ii) In (3), the harmonicity of $v$ may be replaced by the following: $v$ is of $C^{(1)}$ on $\bar{V}$. In (4) the harmonicity of $\omega$ may be replaced by the following: $\omega$ is of $C^{(1)}$ and closed on $\bar{V}$.

(iii) If $V^{\prime} \subset V$ then

$$
L_{0 V^{\prime}}\left(L_{0 V} f\right)=L_{0 V} f, \quad L_{1 V^{\prime}}\left(L_{1 V} f\right)=L_{1 V} f
$$

on $V^{\prime}$ for any $\mathrm{f}$ on $\alpha$; here the subscripts $V^{\prime}$ and $V$ express the region where the operators are considered.

(iv) Conversely, let $V_{1}, \cdots, V_{n} \subset V$ be mutually disjoint and such that $V-\cup_{k=1}^{n} V_{k}$ is relatively compact. Given $f$ on $\alpha$, suppose a $u$ on $V$ satisfy (2) and

$$
u=L_{0 V_{k}} u \quad\left(u=L_{1 V_{k}} u\right)
$$

on $V_{k}, k=1, \cdots, n$. Then $u=L_{0} f\left(u=L_{1} f\right.$, resp.) on $V$.

3. We find in Ahlfors-Sario [1, p. 176ff] that $\varphi_{\Omega}$ and $\phi_{\Omega}$ are characterized as functions regular on $\Omega-\{\infty\}$, having expansion (1) about $\infty$, and such that

$$
L_{0}\left(\operatorname{Re} \varphi_{\Omega}\right)=\operatorname{Re} \varphi_{\Omega}, \quad L_{1}\left(\operatorname{Re} \phi_{\Omega}\right)=\operatorname{Re} \phi_{\Omega}
$$

on $\partial \Omega$; this means the validity of (5) on $V_{1}, \cdots, V_{n}$ with compact $\Omega-\cup_{k=1}^{n} V_{k}$, which 
is independent of the choice of $V_{k}$ because of the above remarks (iii) and (iv). Therefore

THEOREM 1. A region $\Delta$ in the $z=x+$ iy-plane with $\infty \in \Delta$ is a minimal horizontal (vertical) parallel slit plane if and only if

$$
L_{0} x=x \quad\left(L_{1} x=x, \text { resp. }\right)
$$

on $\partial \Delta$.

It is evident that the condition is equivalent with

$$
L_{1} y=y \quad\left(L_{0} y=y, \text { resp. }\right) .
$$

On regarding the definition of $L_{0}$ we see that the validity of $L_{0} x=x$ on a $V$ is equivalent with the following: $\iint_{V}(\partial v / \partial x) d x d y=\int_{\alpha} v d y$. Consequently a region $\Delta$ with $\infty \in \Delta$ is a minimal horizontal parallel slit plane if and only if

$$
\iint_{\Delta} \frac{\partial h}{\partial x} d x d y=0
$$

for every $h$ hich is of $C^{(1)}$ in $\Delta$, vanishes identically in a neighborhood of $\infty$, and has finite $D_{\Delta}(h)$. This is nothing but the original characterization of minimality due to Koebe [4].

From Theorem 1 and remarks (iii), (iv) of $2^{\circ}$, we obtain the following which is Theorem 12 of Suita [8]:

THEOREM 2. Let $\infty \in \Delta_{k}(k=1, \cdots, n)$ have mutually disjoint $\Delta_{k}^{C}$, and let $\Delta=\cap_{k=1}^{n} \Delta_{k}$. Then $\Delta$ is a minimal horizontal (vertical) parallel slit plane if and only if so are all the $\Delta_{k}$.

4. Circular and radial slit planes are characterized by $L_{0}$ and $L_{1}$ in the similar way. Slit disks and annuli are the same if the outer (and inner) periphery is assumed to be isolated from other part of the boundary. For example

Let $\Delta$ be a circular slit annulus with inner and outer radius $0<Q^{\prime}$ and $Q<\infty$, respectively. Let $\left(|z|=Q^{\prime}\right)$ and $(|z|=Q)$ be isolated from $E=\Delta^{C} \cap\left\{z\left|Q^{\prime}<\right| z \mid<Q\right\}$. Then $\Delta$ is a minimal circular slit annulus if and only if $L_{1}(\log |z|)=\log |z|$ on $E$.

The change of the independent variable in (4) implies the following, which is contained in Theorem 11 of Suita [8]:

TheOREM 3. Let a circular slit annulus $\Delta$ and its slits $E$ be as above. Let $\Delta^{\prime}$ be a horizontal parallel slit plane such that $E^{\prime}=\Delta^{\prime C}$ is contained in the interior of a vertical parallel strip with width $2 \pi$. Suppose that $E$ is the image of $E^{\prime}$ under the mapping $z \rightarrow \exp i z$. Then $\Delta$ is minimal if and only if $\Delta^{\prime}$ is minimal.

5. Characterizing minimal circular slit annuli by extremal length is easier than that of parallel slit plane. The former is found in, e.g., Reich-Warschawski [5] (for slit disk, though) or Sakai [7], and the latter is in Jenkins [3] as we have mentioned. 
The former is as the following:

Let $\Delta$ be as in $4^{0}$. Let $\Gamma$ be the family of all the closed rectifiable curves in $\Delta$ separating the inner and outer peripheries. Then $\Delta$ is minimal if and only if $\log \left(Q / Q^{\prime}\right)=2 \pi / \lambda(\Gamma)$.

The following is derived from this:

THEOREM 4. Let $\Delta$ be a plane region containing $\infty$. Let $R$ be a rectangle whose interior contains $\Delta^{C}$ and sides are parallel to the coordinate axes. Let $a$ and $b$ be respectively the width and the height of $R$. Let $\Gamma$ be the family of all the rectifiable curves in $R \cap \Delta$ joining the both vertical sides of $R$. (i) If $\Delta$ is minnal, then $\lambda(\Gamma)$ $=a / b$ for any $R$; (ii) If there exists an $R$ with $\lambda\left(I^{\prime}\right)=a / b$, then $\Delta$ is minimal.

Concerning (ii), Jenkins [3] assumed the validity of $\lambda(\Gamma)=a / b$ for all sufficiently large square $R$. The present form the characterization by moduli of quadrilaterals is stated without proof by Grötzsch [2, p. 188]. The above is Theorem 8 of Suita [8].

Proof. (i) With the aid of linear transformation, we may assume in advance that $a=2 \pi$. Map $R$ by $\zeta=$ const $\cdot \exp i z$ onto $1<|\zeta|<\exp b$ and let the image of $\Delta^{C}$ be $\tilde{E}$. By Theorem $3 \tilde{\Delta}=(1<|\zeta|<\exp b)-\tilde{E}$ is minimal, so that $b=2 \pi / \lambda(\tilde{\Gamma})$, where $\tilde{\Gamma}$ is the family of all the closed curves in $\tilde{J}$ separating the inner and outer peripheries. From the general theory of extremal length, it is easy to obtain $2 \pi / b \leqq \lambda(\Gamma), \lambda(\Gamma) \leqq \lambda(\tilde{\Gamma})$. Thus $\lambda(\Gamma)=2 \pi / b$.

(ii) We may assume in advance that $a=\pi$. Let $\hat{R}$ and $\hat{E}$ be obtained from $R$ and $E$, respectivey, by the reflection across the right vertical side of $R$. Let $\hat{\Gamma}$ be the family of curves obtained from $I^{\prime}$ by the same reflection. Map $\hat{R}$ by $\zeta=$ const $\exp i z$ onto $1<|\zeta|<\exp b$ and let the image of $\hat{E}$ be $\tilde{E}$. Consider $\tilde{\Delta}$ and $\tilde{\Gamma}$ as before. From the general theory, we have $2 \pi / b \leqq \lambda(\tilde{\Gamma}), \lambda(\tilde{\Gamma}) \leqq \lambda(\hat{\Gamma}), \lambda(\hat{\Gamma})=2 \lambda(\Gamma)$. Thus, by the assumption, $b=2 \pi / \lambda(\tilde{\Gamma})$, and, therefore, $\tilde{\Delta}$ is minimal. By Theorem 3 $\hat{E}^{c}$ is minimal, so that, by Theorem $2, \Delta$ is minimal.

\section{REFERENCES}

[1] Ahlfors, L. V., AND L. Sario, Riemann surfaces. Princeton Univ. Press, 1960.

[2] Grötzsch, H., Zum Parallelschlitztheorem der konformen Abbildung schlichter unendlich-vielfach zusammenhängender Bereiche. Leipziger Berichte 83 (1931), $185-200$.

[ 3 ] Jenkins, J. A., Univalent functions and conformal mapping. Springer-Verlag., 1958.

[4] Koebe, P., Zur konformen Abbildung uendlich-vielfach zusammenhängender schlichter Bereiche auf Schlitzbereiche. Gött. Nachr. (1918).

[5] Reich, E., AND S. E. WARSchaWski, On canonical conformal maps of regions of arbitrary connectivity. Pacific J. of Math. 10 (1960), 965-985.

[6] SARIO, L., A linear operator method on arbitrary Riemann surfaces. Trans. Amer. Math. Soc. 72 (1952), 281-295.

[7] Sakai, A., On minimal slit domains. Proc. Japan Acad. 35 (1959), 128-133.

[8] Suita, N., Minimal slit domains and minimal sets. Ködai Math. Sem. Rep. 17 (1965), 166-186.

College of General Education, University of Tokyo. 